\title{
El codiseño como impulso del compromiso del estudiantado universitario
}

\author{
Anna Escofet Roig \\ Universitat de Barcelona \\ mail: annaescofetaub.edu \\ ORCID: https://orcid.org/0000-0002-2230-8802
}

\author{
Ana Novella Cámara \\ Universitat de Barcelona \\ mail: anovelladub.edu \\ ORCID: https://orcid.org/0000-0001-5965-8809
}

\author{
Victoria Morín Fraile \\ Universitat de Barcelona \\ mail: victoriamorinflub.edu \\ ORCID: https://orcid.org/0000-0002-7800-8091
}

\section{RESUMEN}

La relación de partenariado entre estudiantes y docentes universitarios mediante prácticas de codiseño fomenta la participación activa, posibilita el compromiso académico y tiene un valor formativo clave al desarrollar una conciencia metacognitiva sobre lo que se está aprendiendo. El objetivo de la investigación ha sido analizar las representaciones y los marcos significativos de docentes que impulsaron prácticas de codiseño en una universidad pública española. Se implicó a 265 estudiantes de ocho grados universitarios y 10 docentes. Los procesos de codiseño realizados tuvieron que ver con aspectos centrados en los contenidos, la evaluación y las metodologías pedagógicas en asignaturas de distinta tipología. Los resultados permiten presentar cuatro marcos representacionales significativos que los docentes implicados desarrollaron en experiencias de codiseño a partir de la técnica del grupo de discusión. Los hallazgos reafirman las cualidades del codiseño y proponen nuevas claves en relación a su concepción como proceso de construcción participativa, sumando el papel del estudiante como constructor de cambios. Además, proponen dos cualidades nuevas en su caracterización, configurando una propuesta de cinco cualidades: Respeto, Reciprocidad, Responsabilidad, Reflexión y Revisión. Estas aportaciones son clave para acompañar el desarrollo del compromiso del estudiante en la universidad y en su formación.

Palabras Clave: Codiseño; compromiso; aprendizaje; estudiante; participación.

\section{Co-design as an impulse for university students' engagement}

\section{ABSTRACT}

The partnership between students and university teachers through co-design practices encourages active participation, enables academic engagement, and introduces training values when developing the metacognitive awareness of what is being learned. The aim of our research was to analyse representations and significant frameworks of teachers who promoted co-design practices in a Spanish public university. For the purpose of this study, 265 students were involved - from eight different degree programs - and ten teachers. The co-design processes carried out were related to aspects focused on content, assessment, and teaching methodologies in different types of lectures and matters. The results allow us to present four significant frameworks developed by the teachers involved in codesign experiences based on the discussion group technique. The results reaffirm the qualities of co-design and propose new keys in relation to its conception as a participatory construction process, adding the role of the student as a builder of change. In addition, they propose two new qualities in its characterisation, thus configuring a proposal of five qualities: Respect, Reciprocity, Responsibility, Reflection and Revision. These contributions are key to accompanying the development of students' engagement at the university and in their training.

Keywords: Co-design; engagement; learning; student; participation.

ISSN: 0210-2773

DOI: https://doi.org/10.17811/rifie.50.4.2021.825-832 


\section{Introducción}

Una de las preocupaciones del profesorado universitario es conseguir que los estudiantes se sientan motivados en las clases, comprometidos con sus aprendizajes y, también, con la propia institución universitaria. Ello no es una tarea fácil ni tiene una única manera de ser abordado, pero existen evidencias que muestran diversas posibilidades. Algunas investigaciones se centran en la mejora de aspectos conductuales, como la atención, el esfuerzo o la finalización de tareas (Fredricks, Blumenfeld y Paris, 2004; Krause y Coates, 2008) para lograr un aprendizaje efectivo. Otras destacan la importancia de la incorporación de las tecnologías digitales (Alqurashi, 2020; Chena, Lambertb y Guidryb, 2009; Howard y Jie Yang, 2016; Robinson y Hullinger, 2008) en la consecución de la motivación en las aulas. También merecen destacarse las investigaciones que se refieren al papel del docente (Harbour, Evanovich, Sweigart y Hughes, 2015) para lograr el compromiso del estudiantado. Además, claro está, de la importancia de que sea el propio estudiante, con su actitud, quien se comprometa con la institución (Kim, Hong y Song, 2018).

\section{Estudiantes y docentes como partners en la educación superior}

A partir de la relación que se establece entre profesorado y estudiantado, al posibilitar la generación de un ambiente educativo positivo y fomentar el desarrollo emocional, social e intelectual, se puede conseguir un entorno de aprendizaje que fomente la participación (Cooper y Brna, 2002; Munns y Woodward, 2006). De manera concreta, considerar al estudiante como partner hace aflorar una vía a profundizar en la educación superior para implicar al estudiantado en la mejora y el compromiso académico.

La relación de partenariado entre estudiantes y docentes se puede definir como un proceso recíproco de colaboración en el que ambos contribuyen a los procesos de gobernanza en la institución universitaria con el fin de conseguir el cambio, la mejora y la innovación pedagógica. Ello implica diversos niveles de participación, desde la conceptualización pedagógica o la toma de decisiones en relación a una asignatura hasta los procesos participativos para la mejora de un grado universitario. Además, puede implicar a un solo estudiante, a un grupo o a toda la cohorte (Bovill y Bulley, 2011; Bovill, Cook-Sather, Felten, Millard y Moore-Cherry, 2016; Buckley, 2014; Cook-Sather, Bovill y Felten, 2014; Healey, Flint y Harrington, 2014).

Este enfoque tiene una triple potencialidad (Bovil, 2014; Bovill y Bulley, 2011; Cook-Sather et al., 2014). En primer lugar, fomenta una participación activa del estudiantado universitario, tanto en relación con su aprendizaje como en relación al papel que pueden tener en la institución universitaria. En segundo lugar, en el diálogo que se establece entre el punto de vista del estudiantado y la perspectiva del profesorado, ambos pueden comprender(se) y modificar sus perspectivas en relación a la propia institución. Finalmente, que el estudiante se reconozca a sí mismo y reconozca su voz como fuente de cambio tiene un valor formativo clave al fomentar la reflexión a lo largo de todo el proceso y, como consecuencia, desarrollar una conciencia metacognitiva sobre lo que se está aprendiendo.

Para que ello tenga lugar, la relación de partenariado se centra de manera necesaria en la cocreación,

Un proceso de colaboración y reciprocidad a través del cual todos los participantes tienen la oportunidad de contribuir por igual, aunque no necesariamente de la misma manera, a la conceptualización curricular o pedagógica, a la toma de decisiones, a la aplicación, a la investigación o el análisis (Cook-Sather et al., 2014, p.6-7).

Los estudiantes pueden tener diferentes papeles en el proceso de cocreación (Bovill et al., 2016):

- consultor, cuando comparten, analizan y discuten sus perspectivas sobre la enseñanza y el aprendizaje

- coinvestigador, cuando realizan tareas de investigación en el marco de una asignatura o una temática

- codiseñador, cuando diseñan actividades de aprendizaje y evaluación

- representante, cuando sus voces contribuyen a la toma de decisiones en el marco institucional.

Es importante que cualquier institución universitaria se comprometa a la promoción de la cocreación, dado que "la voz de los estudiantes es en sí misma un proyecto de responsabilidad ética" (Taylor y Robinson, 2009, p.71). Una de las formas de cocreación es el codiseño. Esta modalidad de creación posibilita, entre otras cuestiones, que docentes y estudiantado mejoren de manera conjunta la docencia, el aprendizaje y la institución universitaria. Es por ello que es necesario indagar en otros elementos de valor pedagógico que caracterizan al codiseño en la enseñanza superior.

\section{El valor del codiseño en la relación de partenariado}

El codiseño se basa y se integra en las metodologías de participación ciudadana (Brown, 2008; Brown y Wyatt, 2010). El concepto de codiseño aplicado a la educación es relativamente nuevo y tiene que ver con acciones de creatividad colectiva y creación conjunta de conocimiento (Gros, 2019). Su objetivo último es dar poder a los estudiantes en sus procesos de aprendizaje, concibiéndolos como agentes activos que no solo participan en actividades de aprendizaje, sino que también las diseñan y revisan críticamente. Es por ello que es una actividad social que no puede ser separada del contexto en que se desarrollan o participan las personas.

Introducir prácticas de codiseño en las aulas universitarias implica reconocer la naturaleza política de la educación, la necesidad de desafiar las formas de pensar y actuar en las aulas (Bovill, Aitken, Hutchison, Morrison, Roseweir, Scott y Sotannde, 2010), reconocer al estudiantado como interlocutor válido, un individuo activo que en un marco relacional basado en la horizontalidad tiene mucho que decir y aportar a la relación educativa. Ello implica modificar la concepción del mismo acto educativo, que pasa de basarse en la transmisión de conocimiento a centrarse en la producción conjunta del mismo.

Para que ello suceda, es necesario que la institución universitaria permita y facilite el cambio de foco. De manera concreta, ello implica repensar espacios, tiempos y lugares. De hecho, sin el apoyo de la institución, las prácticas de codiseño pueden quedar extremadamente limitadas a experiencias concretas, circunscritas a una materia y a un docente y sin posibilidad de ser reproducidas ni escalables.

En este sentido, es posible destacar las cualidades que debe tener el codiseño en una relación de partenariado (Matthews, 2017):

- Fomentar una relación inclusiva

- Fomentar las relaciones de intercambio de poder a través del diálogo y la reflexión

- Entender la relación de partenariado como un proceso con resultados inciertos

- Participar desde una mirada ética

- Promulgar la relación de partenariado para la transformación 
Además, el docente tiene un papel fundamental (Bovill, Felten y Cook-Sather, 2014) ya que la relación de partenariado implica reconocer la diferencia a la vez que valorar perspectivas y conocimientos. Para ello, es recomendable que la participación del estudiantado sea voluntaria y empezar siempre clarificando los propósitos y la naturaleza del trabajo conjunto a desarrollar. Del mismo modo, es crucial crear objetivos compartidos, asegurando que docentes y estudiantes se reconocen a sí mismos y reconocen la relación de partenariado como legítima.

Por todo ello, en este artículo se presenta una investigación cuyo objetivo ha sido analizar las representaciones y los marcos significativos de los docentes que impulsaron prácticas de codiseño para fomentar el compromiso académico del estudiantado universitario.

\section{Método}

La investigación se ha realizado siguiendo un enfoque metodológico cualitativo, enmarcado en el paradigma sociocrítico y adoptando la opción metodológica de la investigación basada en el diseño (Barab y Squire, 2004; Design-Based Research Collective, 2003), con combinación de métodos mixtos (Wood y Smith, 2018). Esta opción metodológica permite involucrar a todos los participantes desde los momentos iniciales de la investigación y es, en este sentido, la que se adecua de manera más coherente con el marco teórico y con el objetivo formulado. El diseño de la investigación es iterativo, situado, y dirigido a la intervención, pero, simultáneamente, fundamentado en la teoría.

\section{Contexto de estudio}

La investigación se desarrolló durante los meses de octubre a junio del curso académico 2018-19. A lo largo de la misma estuvieron involucrados docentes de diversos ámbitos de conocimiento (Biología, Educación Social, Enfermería, Maestro de Educación Infantil y de Educación Primaria, Pedagogía, Psicología y Trabajo Social), con docencia en asignaturas de distinta tipología (obligatorias, optativas, practicum y créditos de reconocimiento). Los procesos de codiseño realizados tuvieron que ver con aspectos centrados en los contenidos, la evaluación y las metodologías pedagógicas de las asignaturas implicadas.

Concretamente se desarrollaron 8 prácticas de codiseño que implicaron a 265 estudiantes y 10 docentes. El codiseño siempre seguía el mismo proceso. En primer lugar, al inicio del semestre, se presentaba al estudiantado el objetivo a conseguir a través del proceso de codiseño y se invitaba a participar a las personas interesadas. Se explicaba que la participación no sería evaluada y que, por tanto, no sería cuantificada para la calificación de la asignatura. Pero, a la vez, se remarcaba que los resultados del codiseño servirían de base para la mejora de los aspectos codiseñados $\mathrm{y}$, por tanto, sería útil para los compañeros del siguiente semestre. Una vez reclutados los estudiantes se llevaba a cabo el codiseño, cuyo proceso era analizado por el profesorado y los estudiantes implicados para ajustarlo y alcanzar los objetivos definidos colaborativamente. En el proceso se generaron guías de observación y seguimiento.

La investigación se ha desarrollado en un ciclo continuo e iterativo de diseño de la intervención, análisis y rediseño. Los docentes participantes realizaron reuniones periódicas a lo largo de los procesos de codiseño con la finalidad de 1) Preparar y definir cada fase del proceso a partir de las intencionalidades compartidas, 2) Revisar el desarrollo de los codiseños, ajustando el proceso a las posibles incidencias y velando para orientarlo desde los referentes definidos y 3) Realizar el cierre de los procesos de codiseño y evaluar los resultados conjuntamente con los estudiantes mediante un cuestionario y sesiones de valoración.

\section{Participantes, técnica de muestreo y reclutamiento}

Dada la exigencia del diseño de la investigación propuesto, la técnica de muestreo fue intencional. El profesorado implicado en el estudio se seleccionó de forma intencional, al tratarse del equipo investigador y ser los responsables de las asignaturas que participaron en los procesos de codiseño. La tabla 1 muestra el perfil de los docentes participantes.

Tabla 1

Perfil de los docentes participantes

\begin{tabular}{|c|c|c|c|c|c|c|}
\hline Profesorado (categoría) & Perfil & Grado & Título de la asignatura & $\begin{array}{l}\text { Tipología de } \\
\text { la asignatura }\end{array}$ & Tipo de codiseño & $\begin{array}{l}\text { Número de estudiantes } \\
\text { implicados }\end{array}$ \\
\hline Profesor (asociado) & D1 & \multirow[t]{2}{*}{ Enfermería } & \multirow{2}{*}{$\begin{array}{l}\text { Prácticum Comuni- } \\
\text { tario }\end{array}$} & \multirow[t]{2}{*}{ Obligatoria } & \multirow{2}{*}{$\begin{array}{l}\text { Contenido y evaluación } \\
\text { de un seminario }\end{array}$} & \multirow[t]{2}{*}{18} \\
\hline Profesora (titular) & D2 & & & & & \\
\hline Profesora (titular) & D3 & Biología & Desarrollo Sostenible & Obligatoria & $\begin{array}{l}\text { Contenido de tutorías y } \\
\text { evaluación }\end{array}$ & 70 \\
\hline Profesor (titular) & D4 & Trabajo Social & Arte y Trabajo Social & Optativa & $\begin{array}{l}\text { Definición, seguimiento } \\
\text { y evaluación del plan } \\
\text { docente }\end{array}$ & 36 \\
\hline Profesora (titular) & D5 & Pedagogía & $\begin{array}{l}\text { Axiología y Educación } \\
\text { en Valores }\end{array}$ & Obligatoria & $\begin{array}{l}\text { Aplicación, valoración } \\
\text { y rediseño del guion de } \\
\text { un trabajo }\end{array}$ & 24 \\
\hline Profesor (titular) & D6 & $\begin{array}{l}\text { Educación } \\
\text { Social }\end{array}$ & $\begin{array}{l}\text { Acción Socioeduca- } \\
\text { tiva en los Servicios } \\
\text { Sociales }\end{array}$ & Optativa & $\begin{array}{l}\text { Revisión de los criterios } \\
\text { y evidencias de evalua- } \\
\text { ción continuada }\end{array}$ & 45 \\
\hline Profesora (titular) & D7 & $\begin{array}{l}\text { Educación } \\
\text { Social }\end{array}$ & $\begin{array}{l}\text { Animación Sociocul- } \\
\text { tural y Educación en } \\
\text { el Tiempo Libre }\end{array}$ & Obligatoria & $\begin{array}{l}\text { Optimización de las } \\
\text { orientaciones e instru- } \\
\text { mentos de evaluación }\end{array}$ & 6 \\
\hline
\end{tabular}




\begin{tabular}{|l|l|l|l|l|l|l|}
\hline Profesorado (categoría) & Perfil & Grado & Título de la asignatura & $\begin{array}{l}\text { Tipología de } \\
\text { la asignatura }\end{array}$ & Tipo de codiseño & $\begin{array}{l}\text { Número de estudiantes } \\
\text { implicados }\end{array}$ \\
\hline
\end{tabular}

\begin{tabular}{|l|l|l|l|l|l|l|}
\hline Profesora (titular) & D8 & $\begin{array}{l}\text { Pedagogía, } \\
\text { Educación So- } \\
\text { cial, Educación } \\
\text { Infantil, Educa- } \\
\text { ción Primaria }\end{array}$ & $\begin{array}{l}\text { Proyectos de Aprendi- } \\
\text { zaje-Servicio }\end{array}$ & $\begin{array}{l}\text { Créditos de } \\
\text { reconoci- } \\
\text { miento }\end{array}$ & $\begin{array}{l}\text { Seguimiento y evalua- } \\
\text { ción de los aprendizajes }\end{array}$ & $\begin{array}{l}15 \\
\text { Profesora (titular) }\end{array}$ D9 \\
\hline Profesora (catedrática) & D10 & Psicología & $\begin{array}{l}\text { Lenguaje y Comuni- } \\
\text { cación }\end{array}$ & Obligatoria & $\begin{array}{l}\text { Contenido y evaluación } \\
\text { de un seminario }\end{array}$ & 51 \\
\hline
\end{tabular}

Fuente: Elaboración propia

Procedimiento para la obtención y el análisis de la información

Para obtener los marcos representacionales de los docentes implicados se optó por la técnica del grupo de discusión (Boddy, 2005; Onwuegbuzie, Leech, Dickinson y Zoran, 2011), que permitió adentrarnos en los significados que los docentes tenían de su experiencia de acompañar el aprendizaje de los estudiantes mediante la práctica de codiseño después de valorarla conjuntamente con estos. En el grupo de discusión participaron 9 de los 10 profesores implicados, y tuvo una duración de 90 minutos. Para facilitar la discusión y mantener la coherencia se idearon preguntas desencadenantes clave antes de la discusión, relacionadas con las características del proceso de codiseño, la percepción de la experiencia de los estudiantes, la valoración de la experiencia desde la perspectiva docente y la existencia de elementos facilitadores y obstaculizadores. Estas preguntas contenían los elementos conceptuales característicos de los procesos de codiseño identificados en la literatura.

Se realizó la grabación del grupo de discusión para su transcripción y análisis. El análisis de los datos cualitativos obtenidos se realizó siguiendo el procedimiento del análisis del contenido. Se realizó un análisis preliminar de los datos, construyendo un árbol de categorías que recogía los conceptos clave de los procesos de codiseño indicados en la literatura. A partir de ello se realizó un proceso de análisis y categorización de los datos hasta la saturación de la información. Este proceso se realizó en un primer momento por cada investigador y en una segunda revisión por otro investigador del equipo; de esta manera se aseguraba el rigor en la categorización y el análisis de los datos, de forma que permitía afinar la interpretación compartida de la información. El análisis de los datos se realizó de forma manual, sin la utilización de soporte técnico.

\section{Análisis de resultados}

Los resultados obtenidos permiten presentar cuatro marcos significativos que emergen entre los docentes que impulsaron prácticas de codiseño para fomentar el compromiso académico del estudiantado universitario. Estos marcos apuntan aspectos clave para concebir dichas prácticas como oportunidades para promover el compromiso, la implicación y la motivación de los estudiantes, los invita a pensarse como agentes implicados en procesos de aprendizaje y a reflexionar críticamente sobre ellos favoreciendo así su consciencia, implicación y responsabilidad con su formación.

La siguiente tabla muestra el árbol de categorías que permite organizar este apartado de resultados.

Tabla 2.

Marcos significativos de los docentes

\begin{tabular}{|l|l|}
\hline Categoría & Subcategoría \\
\hline El codiseño como forma de aprender & Como estrategia \\
& Como medio \\
& Como secuencia de acciones estratégicas \\
& Como contenido competencial \\
\hline Finalidades del codiseño según el foco de & En los procesos de pensamiento del estudiante \\
incidencia & En la revisión del rol de estudiante de forma consciente \\
\hline Principios nucleares del codiseño & En el aprendizaje competencial como profesional en formación \\
\hline Estrategias para desarrollar procesos de & Proceso de construcción participativa \\
codiseño & Espacio relacional de partenariado \\
& Proceso que intensifica la reflexión y la revisión \\
& Se aprende corresponsabilizándose \\
& La relación comunicativa fortalece el reconocimiento y el autoconocimiento \\
\hline
\end{tabular}

Fuente: Elaboración propia 
A continuación, se ahonda en el análisis de estos marcos significativos, con el detalle de cada categoría y subcategoría.

\section{El codiseño como forma de aprender}

Los participantes en el grupo de discusión coinciden en conceptualizar el codiseño como una forma de aprender. Pero esta manera de aprender es concretada con diferentes etiquetas que utilizan los informantes en el desarrollo de la discusión: elemento, factor, herramienta, procedimiento, espacio, estrategia, propuesta, proceso, oportunidad, experiencia, vivencia, proyecto o construcción.

Si se aproximan los marcos de significado que rodean a las diferentes etiquetas se identifican cuatro subcategorías:

- Como estrategia docente que se introduce para promover que los estudiantes se impliquen en una relación formativa que posibilite alcanzar algunos objetivos. Es una herramienta, procedimiento, factor o estrategia al que recurre el docente como facilitador.

«Y pensaba que realmente deviene un elemento o un procedimiento de aprendizaje, que tu crees que es muy valioso» (D8, p.12)

- Como medio que se organiza para facilitar el aprendizaje. Espacio formativo y relacional donde sumergirse con otros en atender un reto académico. En esta situación de aprendizaje pasan cosas y se hacen cosas prácticas conjuntamente: experiencia, vivencia, oportunidad, espacio o propuesta.

«Y depende del aspecto del diseño que se ha tocado, a lo mejor hará que se lo replanteen más. Por ejemplo, los míos, los míos fueron una microexperiencia. Pensad que ha sido una cosa muy puntual y pequeña» (D10, p.4)

- Como secuencia de acciones estratégicas y organizadas que se hacen colaborativamente entre docente y estudiantes. En el proceso del hacer conjunto se enfrentan a la resolución de tareas que necesitan de desarrollo y evolución: proyecto, proceso, construcción o propuesta.

«Creo que eso. Por tanto, un elemento positivo sería esta parte de cómo recibes tú el hecho de ser invitado a un proceso así, cómo te empodera, cómo te sitúa en ello, ¿no?» (D7, p.7)

- Como contenido competencial que centra la intencionalidad formativa en el qué aprender. Los docentes configuran el contenido a aprender centrándose en los cuatro niveles competenciales relacionados con saber (competencia técnica), saber hacer (competencia metodológica), saber estar (competencia participativa) y saber ser (competencia personal). La inmersión pone a hacer cosas, a usar el conocimiento y a transferirlo. El proceso de desarrollo pone al estudiante en situaciones donde dispone de conocimientos, procedimientos y actitudes que usar y desarrollar con el hacer implicativo.

«Lo otro es devenir de esto, la manera de aprender dentro de la asignatura y como contenido de la asignatura..., además me permite conocer un contenido procedimental que yo pueda usar en mi práctica profesional» (D8, p.13)

\section{Finalidades del codiseño según el foco de incidencia}

El grupo de discusión reconoce el codiseño como metodología que busca mejoras, pero alrededor de esta finalidad clásica emergen otras intencionalidades en función de cuál es el foco del avance o mejora. Mayoritariamente el codiseño en la educación superior busca en el hacer conjunto incidir en una asignatura o componentes alrededor de ésta. En las experiencias impulsadas por los diferentes docentes han surgido otras finalidades a partir del reto compartido de incidir en el compromiso del estudiante en su propio aprendizaje.

«Claro, yo parto de la idea previa que ya hay evidencia que los procesos de codiseño tienen una relación directa sobre el compromiso del estudiantado y su propio aprendizaje. $Y$ nosotros lo hemos medio comprobado. Se me hace difícil, siempre que pueda, no hacer codiseño» (D2, p.13)

De alguna forma la significación del codiseño tiene dimensiones complejas que no le diferencian conceptualmente de lo que es, sino que se diferencia por aquella intencionalidad formativa desde la que se organiza, porque es desde aquí que se favorece el pensarse dentro del proceso formativo desarrollando competencias para ello y para potenciar el compromiso. En definitiva, ejercitar la metacognición para ser autor de su proceso formativo y del compromiso hacia este. Las finalidades que emergen en la discusión identifican hasta tres focos de incidencia:

- En los procesos de pensamiento del estudiante. Las experiencias han creado la posibilidad de pensar sobre un contenido diferente como es su propio aprendizaje en un contexto determinado. Se señala la importancia de que esta oportunidad permita participar activamente, así como dar profundidad y solidez a cómo se aprende. De alguna forma en estos procesos se incrementan las estrategias cognitivas del estudiante.

"Aquí das la opción de trabajar metodológicamente de una manera que tiene un impacto muy importante en el pensamiento del estudiante. Y es igual el contenido específico. La experiencia de haber participado activamente en un proceso de este tipo enseña más que el propio contenido en sí» (D2, p.14)

- En la revisión del rol de estudiante de forma consciente. Los procesos que genera el codiseño favorecen ponerse en situaciones novedosas como estudiante. Ser invitado a pensar alrededor de cómo mejorar su implicación en su formación, así como a ser más consciente de su proceso de aprendizaje. Incrementar el ejercicio de metacognición lleva a repensar su rol de estudiante y pensar en cómo ha aprendido y cómo puede regular su proceso de aprendizaje.

«No, no, no es solo metodología, es que es contenido. Es que yo quiero que mis estudiantes, el día de mañana, codiseñen en sus Centros de Atención Primaria... Es decir, lo quiero convertir en un elemento de aprendizaje que permita reflexionar sobre mi rol como estudiante, sobre la universidad. Además, que me permita conocer un contenido procedimental que yo pueda usar en mi práctica profesional» (D8, p.12)

- En el aprendizaje competencial como profesional en formación. El desarrollo competencial en prácticas de codiseño, según las aportaciones de los docentes del grupo de discusión, tiene una proyección en el ejercicio profesional. Ser consciente de estos aprendizajes y de su sentido posibilitaría que el estudiante incorporase el codiseño como práctica a desarrollar en su ejercicio profesional. De alguna forma se enfatiza el codiseño como forma de construir la identidad profesional.

«Entonces, el hecho que su futuro profesional esté relacionado con la participación con la población permite quizás que toda esta experiencia, en relación a todo un proceso, les permite a ellos pensarlo en relación a su futuro profesional» (D6, p.5) 
Principios nucleares del codiseño

En el desarrollo de la discusión emergían alrededor del concepto y de las finalidades ideas que fundamentaban cómo se concebía el marco de la formación académica centrada en el codiseño. Estas ideas configuran lo que podrían ser los principios nucleares que orientan el codiseño que busca incidir en el compromiso del estudiante:

- Proceso de construcción participativa. Este principio engloba tres componentes clave: proceso, construcción y participación, que deben fusionarse desde el reconocimiento al estudiante como integrante activo que lleva a cabo acciones implicativas que le requieren elaborar y evolucionar en su quehacer. En el grupo emerge que se trata de una participación diferente a la que se da habitualmente entre los estudiantes, que requiere más de ellos, que los lleva a tomar parte y les desvela un rol diferente.

«Eso me hace pensar en el proceso de codiseño como un proceso de construcción participativa. Y es este punto de la participación entre iguales para la construcción, en mi caso de una asignatura, cuando el rol clásico es siempre la construcción desde la situación y la ubicación de la autoridad, del conocedor, ¿no?» (D3, p.2)

- Espacio relacional de partenariado que es horizontal, que es inclusivo. Los informantes constatan que en la experiencia de codiseño se ha establecido una relación diferente con los estudiantes y se les saca de su rol de actividad distante a una relación de proximidad que en el hacer colaborativo les iguala. Se destaca que favorece una apertura recíproca docente-estudiantes y estudiantes-docentes. Se menciona reiteradamente que trabajar juntos docente y estudiantes deforma los roles clásicos y favorece la revisión de la autoimagen como estudiante.

«Sería esta analogía aplicada a cualquier actividad y ahora aplicada a la enseñanza, a esta construcción entre todos, en igualdad de condiciones, que es esta cosa tan valiosa que tiene y que también implícitamente seguro que está en el respeto, responsabilidad y reciprocidad, ¿no? Repartir ¿no? Y es indispensable para poder hacer este proceso participativo, porque si no, no se puede construir ¿no?» (D3, p.2)

- Proceso que intensifica la reflexión y la revisión como estrategias metacoginitivas. La reflexión como catalizador permite pararse a pensar en las estrategias que han facilitado la emergencia de contenidos para iniciar el codiseño. Pero en este hacer el docente centra e incrementa la reflexión y revisión alrededor de cómo han aprendido, qué han aprendido y en qué les ha incidido (explora el ser y estar). Favoreciendo la toma de consciencia de cómo se aprende a aprender y adentrándose en un ejercicio de metacognición.

«Un proceso de codiseño tiene una parte de metacognición, de repensar. Y puedo repensar sobre la evaluación, poner el foco en la evaluación y tu papel, la transferencia de esto a otras situaciones» (D7, p.4)

- Se aprende corresponsabilizándose. En el grupo emerge la idea de que implicar al estudiante en la toma de decisiones alrededor de cuestiones vinculadas al proceso de docencia y aprendizaje moviliza el compromiso y la responsabilidad. Estar inmerso en ese análisis del proceso de aprendizaje en colectividad, en colaboración y cooperación conlleva una mayor vinculación en aquello que se cuestiona sintiéndose parte y tomando parte, hecho que incrementa su mirada crítica y compromiso.
«Por eso me aparecía más esta idea como de compromiso en lo que tenemos entre manos... Yo me siento más parte de ello, más directamente vinculado, ¿no? Porque me exige una implicación más directa» (D8, p.2)

- La relación comunicativa fortalece el reconocimiento y autoconocimiento. La comunicación va más allá de la escucha, se fortalece desde el diálogo deliberativo y constructivo en el que se vincula lo relacional, lo emocional y lo afectivo. Mediante la comunicación se anima y se motiva al grupo a seguir y a implicarse. El discurso que se teje en la relación centrada en el codiseño está cargado de mensajes que mediatizan y condicionan, para lo bueno y no tan bueno, el proceso de aprendizaje.

"Quizá hace que ellos perciban que el profesor está más predispuesto a tener una actitud que facilitará que pasen cosas que antes no han pasado. Sí que hace que se genere un clima, una relación con el estudiante que es diferente... de las que yo misma establezco en otras asignaturas que tienen un formato más clásico y tradicional, de clase magistral al uso. La diferencia en esta asignatura es que el clima, la relación que se establece profe-estudiante, por este factor de innovación, es diferente y facilita que haya una comunicación diferente al otro caso» (D3, p.6)

Estrategias docentes para desarrollar procesos de codiseño

Los resultados muestran que el codiseño es una práctica innovadora en la educación superior con entornos y dimensiones diversos, desde modificar algún aspecto específico de una asignatura o repensar el clima en el aula a redefinir una titulación, y sin ninguna duda, fomenta el compromiso académico del estudiantado. A la vez, también puede servir para incorporar nuevos dispositivos pedagógicos o materiales en relación con las necesidades o demandas emergentes de estudiantado y/o profesorado, así como institucionalizar las relaciones de partenariado como un elemento fundamental de la responsabilidad social universitaria.

Los docentes en el grupo de discusión compartían algunas estrategias que han favorecido el desarrollo del codiseño y su énfasis en el compromiso y responsabilidad del estudiante en diferentes focos. Se han identificado cinco estrategias:

- Estrategias de planificación que están presentes de principio a fin del codiseño. Estas tienen la intención de centrar el tema, de tener la aceptación de implicación por parte del estudiante, de buscar y mantener su vinculación, así como de que tome parte de ir ajustando el proceso de codiseño en función de los avances. El proceso que sigue el codiseño se dibuja desde las intencionalidades ajustadas al tiempo que tenemos con un sentido y sentir.

"Creo que hay estrategias de información y de convocatoria, de centrar qué queremos hacer, ¿no? Iniciales, igual que hay estrategias de cierre como decíamos ahora, ¿no? Estrategias que tienen que ver con este reconocimiento y trabajan el autoconcepto o para definir lo que compartimos. También estrategias de convocatoria y para planificar el proceso juntos, de centrar el objeto y de cómo presentamos cada sesión ¿no? Cómo cada sesión tiene momentos para decir lo que trabajamos, cómo lo trabajamos y lo que hemos acordado» (D7, p.9)

- Estrategias de participación. El codiseño requiere de un hacer implicativo que sitúa al estudiante en un hacer participado diferente con una intensidad y relevancia que incide en un estar cercano y comprometido. El docente recurre a estrategias que faciliten esta participación implicativa 
y cooperativa. Incluso en la discusión emerge la idea de diseñar el codiseño o de la coplanificación de este.

«Creo que hay codiseños que necesitan esta coplanificación del diseño, porque si no la verticalidad no se rompe. Creo que esta es una dificultad. Aunque se diga la diferencia entre teoría y práctica, ¿no? Si la constatación que los estudiantes van tomando en el proceso, no verifica que es un codiseño de verdad...» (D5, p.10)

- Estrategias de comunicación. Los informantes señalan cómo cultivar la proximidad entre estudiantes y docente con otras directrices, una de éstas es tener hilo directo mediante canales comunicativos. Compartir las decisiones, elevarlas y comprometernos a ellas pasa por mostrar y constatar comunicativamente que así es.

«Cuando acabamos y había hecho el vaciado de lo que habiamos dicho y tal. Y bueno, digo, ahora, para tenerlo más fresco les envío un correo, les doy las gracias, les digo cuáles han sido sus aportaciones y de qué manera las incorporaré en el guion. La idea que tenía ahora es que cuando empiece en febrero, si aun tengo activa la gente en el campus, les enviaré el documento del nuevo guion que ellos hacen» (D5, p.8)

- Estrategias de reflexión y revisión. Los docentes manifiestan como en el proceso de codiseño han pensado y analizado esta forma de aprender para ir ajustando la ayuda, así como para incidir más en el compromiso de los estudiantes. Siendo un proceso de innovación y de identificación de los elementos que lo hacen posible. El compromiso docente busca conectar con el compromiso del estudiante.

«Y esto que decís, hacer gradaciones de codiseño con diferentes objetivos: menor dedicación, mayor dedicación, más impacto ¿Qué estás dispuesto a hacer? Porque si revisamos lo que hacemos, seguro que lo podemos ordenar con estos criterios. En todos los casos, seguramente estamos satisfechos, ¿no? Y quizá esto servirá para situar y decir: "¿qué puedo hacer yo?"» (D3, p.14)

- Estrategias de visibilización y materialización. Un elemento coincidente entre los participantes en el grupo de discusión es la importancia de hacer visible lo que se está haciendo y mostrarlo, así como subrayar la coproducción con los estudiantes. Es necesario ir mostrando y dar valor a los avances, ser conscientes que se está avanzando es clave.

«A mi se me había ocurrido añadir también otra que sería la visibilización que estamos haciendo, la transparencia que estamos haciendo, que realmente el estudiante vea que tiene una consecuencia visible, que se plasma en un avance o en un cambio en una pequeña innovación concreta, aunque no la disfrute ella o él» (D5, p.1)

\section{Discusión y conclusiones}

La investigación desarrollada se ha centrado en el análisis de los marcos de significado otorgados por docentes a diversas experiencias de codiseño desarrolladas en diversos ámbitos de conocimiento y asignaturas. Estos marcos reafirman aspectos clave para concebir las prácticas de codiseño como oportunidades para promover el compromiso, la implicación y la motivación del estudiantado, así como proponen nuevos avances que refuerzan esta concepción.

El primer aspecto clave está relacionado con la conceptualización de codiseño y una de sus cualidades dentro de una relación de partenariado (Matthews, 2017), que es ser un proceso de construcción participativa. El codiseño tiene a la participación como componente fundamental, porque sin ella no hay proceso ni construcción y porque mediante ella se da la transformación de los implicados en el proceso. Es una participación intencionalmente diferente a la que en esos momentos caracteriza la implicación del estudiantado en la docencia, en el aprendizaje y en la universidad. El estudiante, en estos procesos, toma partido de las transformaciones que se dan, es artífice de los cambios y, así, es su coautor. Por ello, es constructor de aquello que colaborativamente define y de los nuevos escenarios que configura en relación con el aprendizaje. Esta cualidad nos lleva a defender la incorporación del papel de constructor como uno más de los papeles que el estudiante puede tener dentro de un proceso de cocreación (Bovill et al., 2016). Estamos ante el reto, como comunidad universitaria, de dar un giro a la concepción del compromiso en la universidad y en la formación, y a la concepción del estudiante dentro de ella.

El segundo aspecto clave que aporta la investigación es que el codiseño que busca fortalecer el compromiso del estudiante tiene como finalidad el desarrollo de la competencia transversal del compromiso y la corresponsabilidad, que requiere que el docente acompañe procesos donde enseña cómo se aprende y cómo se aprende a aprender a partir de estrategias de metacognición socializada y cooperativa (Gargallo, Pérez-Pérez, García-García, Giménez y Portillo, 2020) donde el estudiante entra en un estado interior para pensar(se) y (re)pensarse como estudiante. Además, la experiencia de codiseño favorece en los estudiantes una imagen positiva de ellos mismos como aprendices comprometidos en su formación que se reconstruye en el mismo proceso de codiseño a partir de las estrategias que le caracterizan, así como elaboran una imagen más cercana y positiva de la función docente e incluso de la institución universitaria.

El tercer aspecto clave ha sido reafirmar que el codiseño tiene como requisitos fundamentales las cualidades de respeto, reciprocidad y responsabilidad (Cook-Sather et al., 2014), condiciones nucleares en cualquier proceso de enseñanza y aprendizaje. Además, la investigación identifica dos cualidades más que serían nuevos requisitos fundamentales, la reflexión y la revisión. En los procesos de codiseño se requiere que los participantes conecten con su propia experiencia para conocer en profundidad el contexto y la práctica educativa que tratan de cambiar, cómo se sitúan y qué sienten al respecto. Reflexión y revisión alimentan el proceso de metacognición que favorece la toma de conciencia del proceso, el uso y desarrollo de sus competencias, los aprendizajes construidos y la transformación personal como estudiante y profesional en formación. El codiseño asegura el paso necesario que va desde la acción a la reflexión, y de la reflexión a la acción de nuevo, lo que permite a los estudiantes conseguir un mayor nivel de comprensión y de conciencia del proceso de aprendizaje y un compromiso más profundo en la mejora de sus resultados (Könings, Seidel y Van Merriënboer, 2014). La investigación ha constatado la reflexión y la revisión como principios nucleares del codiseño, así como finalidades y estrategias fundamentales. Este hallazgo sugiere proponer que el codiseño se caracteriza por cinco cualidades: respeto, reciprocidad, responsabilidad, reflexión y revisión.

Es por ello que se afirma que el codiseño es una metodología fundamental para promover el compromiso, la implicación y la motivación de los estudiantes, al invitarlos a pensarse como tales y a reflexionar críticamente sobre los procesos de enseñanza y aprendizaje. Esta metodología se dota de estrategias para planificar el codiseño, fomentar la participación, promover la comunicación, incrementar la reflexión y la revisión en todo el proceso, así como visibilizar y materializar los avances.

Para concluir, es relevante remarcar el potencial que tiene el codiseño para promover el empoderamiento de los estudiantes 
en relación tanto a su aprendizaje como al papel transformador que pueden ocupar dentro de la institución universitaria. Además, supone en sí mismo un principio de valor que apunta a la responsabilidad social universitaria, poniendo el acento en el compromiso y la voluntad de trabajo conjunto con todos los miembros de la comunidad universitaria.

\section{Referencias bibliográficas}

Alqurashi, E. (ed.). (2020). Handbook of Research on Fostering Student Engagement with Instructional Technology in Higher Education. IGI Global.

Barab, S. y Squire, K. (2004). Design-Based Research: Putting a Stake in the Ground. Journal of the Learning Sciences, 13(1), $1-14$.

Boddy, C. (2005). A rose by any other name may smell as sweet but "group discussion" is not another name for a "focus group" nor should it be. Qualitative Market Research: An International Journal, 8(3), 248-255. Recuperado de https://doi. org/10.1108/13522750510603325

Bovill, C. (2014). An investigation of co-created curricula within higher education in the UK, Ireland and the USA. Innovations in Education and Teaching International, 51(1) 15-25.

Bovill, C; Aitken, G.; Hutchison, J.; Morrison, F.; Roseweir, K.; Scott, A. y Sotannde, S. (2010). Experiences of learning through collaborative evaluation from a masters programme in professional education, International Journal for Academic Development, 15(2), 143-154.

Bovill, C., y Bulley, C.J. (2011). A model of active student participation in curriculum design: exploring desirability and possibility. En Rust, C. (ed.). Improving Student Learning. Global Theories and Local Practices: Institutional, Disciplinary and Cultural Variations. Oxford Brookes University.

Bovill, C., Cook-Sather, A., Felten, P., Millard, L., y Moore-Cherry, N. (2016). Addressing potential challenges in co-creating learning and teaching: Overcoming resistance, navigating institutional norms and ensuring inclusivity in student-staff partnerships. Higher Education, 71(2), 195-208.

Bovill, C.; Felten, P. y Cook-Sather, A. (2014). Engaging Students as Partners in Learning and Teaching (2): Practical guidance for academic staff and academic developers. Conference at International Consortium on Educational Development. Recuperado de https://www.researchgate.net/publication/271200592_Engaging_Students_as_Partners_in_Learning_and_Teaching_2_ Practical_guidance_for_academic_staff_and_academic_developer

Brown, T. (2008). Design thinking. Harvard Business Review, 25, 85-92.

Brown, T., y Wyatt, J. (2010). Design Thinking for Social Innovation. Stanford Social Innovation Review, 8, 31-35.

Buckley, A. (2014). How radical is student engagement? (And what is it for?). Student Engagement and Experience Journal, 3(2). Recuperado de http://dx.doi.org/10.7190/seej.v3i2.95

Cook-Sather, A., Bovill, C. y Felten, P. (2014). Engaging Students as Partners in Learning and Teaching: A guide for faculty. Jossey Bass.

Cooper, B. y Brna, P. (2002). Supporting high quality interaction and motivation in the classroom using the social and emotional learning and engagement in the NIMS project. Education, Communication and Information, 2(4), 113-138.

Chena, D., Lambertb, P. y Guidryb, K.R. (2009). Engaging online learners: The impact of Web-based learning technology on college student engagement. Computers $\mathcal{E}$ Education, 54, 1222-1232.

Design-Based Research Collective. (2003). Design-Based Research: An Emerging Paradigm for Educational Inquiry. Educational Researcher, 32(1), 5-8.

Fredricks, J.A., Blumenfeld, P.C., y Paris, A.H. (2004). School engagement: potential of the concept, state of the evidence. Review of Educational Research, 74(1), 59-109.

Gargallo, B.; Pérez-Pérez, C.; Garcia-Garcia, F.J.; Giménez, J.A., y Portillo, N. (2020). La competencia aprender a aprender en la universidad: propuesta de modelo teórico. Educación XX1, 23(1), 19-44, https://orcid.org/10.5944/educXX1.23367

Gros, B. (2019). La investigación sobre el diseño participativo de entornos digitales de aprendizaje. Universitat de Barcelona. Recuperado de http://hdl.handle.net/2445/144898

Harbour, K. E., Evanovich, L. L., Sweigart, C. A., y Hughes, L. E. (2015). A brief review of effective teaching practices that maximize student engagement. Preventing School Failure, 59(1), 5-13.

Healey, M., Flint, A. y Harrington, K. (2014). Engagement through partnership: Students as partners in learning and teaching in higher education. Higher Education Academy. Recuperado de https://www.heacademy.ac.uk/engagement-throughpartnership-students-partners-learning-and-teachinghigher-education

Howard, S.K. y Jie Yang. J. (2016). Student rules: Exploring patterns of students' computer-efficacy and engagement with digital technologies in learning. Computers $\mathcal{E}$ Education, 101, 29-42.

Kim, H.J., Hong, A.J. y Song, A.D. (2018). The Relationships of Family, Perceived Digital Competence and Attitude, and Learning Agility in Sustainable Student Engagement in Higher Education. Sustainability, 10(12), 1-16.

Könings, K.; Seidel T. y Van Merriënboer, J. (2014). Participatory design of learning environments: integrating perspectives of students, teachers and designers. Instructional Science, 42(1), $1-9$.

Krause, K. y Coates, H. (2008). Students' engagement in firstyear university. Assessment and Evaluation in Higher Education, 33(5), 493-505.

Matthews, K. E. (2017). Five Propositions for Genuine Students as Partners Practice International. Journal for Students as Partners, 1(2), 1-9.

Munns, G. y Woodward, H. (2006). Student engagement and student self-assessment: the REAL framework. Assessment in Education: Principles, Policy \& Practice, 13(2), 193-213.

O'Kane, C. (2000). The development of participatory techniques. Facilitating children's views about decisions that affect them. En P. Christensen \& A. James (Eds), Research with Children (pp. 125-155). Falmer Press.

Onwuegbuzie, A. J., Leech, N. L., Dickinson, W. B., y Zoran, A. G. (2011). Un marco cualitativo para la recolección y análisis de datos en la investigación basada en grupos focales. Paradigmas: Una revista Disciplinar de Investigación, 3(2), 127-157.

Robinson, C.C., y Hullinger, H. (2008). New benchmarks in higher education: Student engagement in online learning. Journal of Education for Business, 84(2), 101-108.

Taylor, C. y Robinson, C. (2009). Student voice: theorising power and participation. Pedagogy, culture and society, 17(2), 161-175.

Wood, P. y Smith, J. (2018). Investigar en educación. Conceptos básicos y metodología para desarrollar proyectos de investigación. Narcea. 